\title{
Determinism and a Supersymmetric Classical Model of Quantum Fields
}

\author{
Hans-Thomas Elze \\ Dipartimento di Fisica, Via Filippo Buonarroti 2, I-56127 Pisa, Italia * \\ Received on 23 February, 2005
}

\begin{abstract}
A quantum field theory is described which is a supersymmetric classical model. Supersymmetry generators of the system are used to split its Liouville operator into two contributions, with positive and negative spectrum, respectively. The unstable negative part is eliminated by a positivity constraint on physical states, which is invariant under the classical Hamiltonian flow. In this way, the classical Liouville equation becomes a functional Schrödinger equation of a genuine quantum field theory. Thus, 't Hooft's proposal to reconstruct quantum theory as emergent from an underlying deterministic system, is realized here for a field theory. Quantization is intimately related to the constraint, which selects the part of Hilbert space where the Hamilton operator is positive. This is seen as dynamical symmetry breaking in a suitably extended model, depending on a mass scale which discriminates classical dynamics beneath from emergent quantum mechanical behaviour.
\end{abstract}

\section{INTRODUCTION}

In a recent letter, I discussed anew the (dis)similarity between the classical Liouville equation and the Schrödinger equation [1]. In suitable coordinates both appear quite similar, apart from the characteristic doubling of the classical phase space degrees of freedom as compared to the quantum mechanical case. The Liouville operator is Hermitian in the operator approach to classical statistical mechanics developed by Koopman and von Neumann [2]. However, unlike the case of the quantum mechanical Hamiltonian, its spectrum is generally not bounded from below. Therefore, attempts to find a deterministic foundation of quantum theory - based on a relation between the Koopman-von Neumann and quantum mechanical Hilbert spaces and equipped with the corresponding dynamics - must particularly answer the question of how to construct a stable ground state.

Investigations of these problems are to a large extent motivated by work of 't Hooft, who has argued in favour of such model building, in order to gain a fresh look at the persistent clash between general relativity and quantum theory [3]. Besides, since its very beginnings, there have been speculations about the possibility of deriving quantum theory from more fundamental and deterministic dynamical structures. The discourse running from Einstein, Podolsky and Rosen [4] to Bell [5], and involving numerous successors, is well known, debating the (im)possibility of (local) hidden variables theories.

Much of this debate has come under experimental scrutiny in recent years. No disagreement with quantum theory has been observed in the laboratory experiments on scales very large compared to the Planck scale. However, the feasible experiments cannot rule out the possibility that quantum mechanics emerges as an effective theory only on sufficiently large scales and can indeed be based on more fundamental models.

In various examples, the emergence of a Hilbert space structure and unitary evolution in deterministic classical models has been demonstrated in an appropriate large-scale limit.

* Permanent address: Instituto de Física, Universidade Federal do Rio de Janeiro C.P. 68.528, 21941-972 Rio de Janeiro, RJ, Brazil
However, in all cases, it is not trivial to assure that a resulting model qualifies as "quantum" by being built on a well-defined groundstate, i.e., with an energy spectrum that is bounded from below.

A class of particularly simple emergent quantum models comprises systems which classically evolve in discrete time steps $[3,6]$. Employing the path integral formulation of classical mechanics introduced by Gozzi and collaborators [8], it has been shown that actually a large class of classical models turns into unitary quantum mechanical ones, if the Liouville operator governing the statistical evolution is discretized [7]. However, there remains a large arbitrariness in such discretizations, which one would hope to reduce with the help of consistency or symmetry requirements of a more physical theory.

Furthermore, it has been observed that classical systems with Hamiltonians which are linear in the momenta are also suitable for a reformulation in quantum mechanical terms. In order to provide a groundstate for such systems, a new kind of gauge fixing or constraints implementing "information loss" at a fundamental level have been invoked $[3,9,10]$. Again, a unifying dynamical principle leading to the necessary truncation of the Hilbert space is still missing.

Various other arguments for deterministically induced quantum features have been proposed recently - see works collected in Part III of Ref. [11], for example, or Refs. [12, 13], concerning statistical and/or dissipative systems, quantum gravity, and matrix models.

Many of these attempts to base quantum theory on a classical footing can be seen as variants of the earlier stochastic quantization procedures of Nelson [14] and of Parisi and $\mathrm{Wu}$ [15], often accompanied by a problematic analytic continuation from imaginary (Euclidean) to real time, in order to describe evolving systems.

In distinction, one may aim at a truly dynamical understanding of the origin of quantum phenomena. In this work, I present a deterministic field theory from which a corresponding quantum theory emerges by constraining the classical dynamics. In particular, I will extend the globally supersymmetric ("pseudoclassical") onedimensional model introduced in Ref. [1] to field theory. Thus, a functional Schrödinger equation is obtained with a positive Hamilton operator, involving the standard scalar boson part in the noninteracting case. 
Key ingredient is a splitting of the phase space evolution operator, i.e., of the classical Liouville operator, into positive and negative energy contributions. The latter, which would render the to-be-quantum field theory unstable, are eliminated by imposing a "positivity constraint" on the physical states, employing the Koopman-von Neumann approach $[2,16]$. The splitting of the evolution operator and subsequent imposition of the constraint makes use of the supersymmetry of the classical system, which furnishes Noether charge densities which are essential here. While, technically, this is analogous to the imposition of the "loss of information" condition in 't Hooft's and subsequent work $[3,9,10]$, it is hoped that the present extension towards interacting fields opens a way to better understand the dynamical origin of such a constraint. While a dissipative information loss mechanism is plausible, alternatively a dynamical symmetry breaking may be considered as the cause.

This paper is organized as follows. In Section II, the (pseudo)classical field theory is introduced and its equations of motion and global supersymmetry derived. Section III is devoted to the statistical mechanics of an ensemble of such systems, its Hilbert space description and Liouville equation, in particular. The Liouville equation is then cast into the form of a functional Schrödinger equation in Section IV. Also the necessary positivity constraint on physical states is discussed, constructed, and incorporated there which turns the emergent Hamiltonian into a positive local operator with a proper quantum mechanical groundstate. In the concluding Section V, I mention some interesting topics for further exploration, especially the relation of the positivity constraint to symmetry breaking.

\section{THE SUPERSYMMETRIC CLASSICAL MODEL}

The following derivation will newly make use of "pseudoclassical mechanics" or, rather, pseudoclassical field theory. These notions have been introduced through the work of Casalbuoni and of Berezin and Marinov, who considered a Grassmann variant of classical mechanics, studying the dynamics of spin degrees of freedom classically and after quantization in the usual way [17].

Classical mechanics based on Grassmann algebras has more recently found much attention in attempts to better understand the zerodimensional limit of classical and quantized supersymmetric field theories, see Refs. [18, 19] and further references therein.

Let us introduce a "fermionic" field $\psi$, together with a real scalar field $\phi$. The former is represented by the nilpotent generators of an infinite dimensional Grassmann algebra [20]. They obey:

$$
\left\{\psi(x), \psi\left(x^{\prime}\right)\right\}_{+} \equiv \psi(x) \psi\left(x^{\prime}\right)+\psi\left(x^{\prime}\right) \psi(x)=0,
$$

where $x, x^{\prime}$ are coordinate labels in Minkowski space. All elements are real.

Then, the classical model to be studied is defined by the action:

$$
S \equiv \int \mathrm{d}^{4} x\left(\dot{\phi} \dot{\psi}-\phi\left(-\Delta+m^{2}+v(\phi)\right) \psi\right) \equiv \int \mathrm{d} t L,
$$

where dots denote time derivatives, and $v(\phi)$ may be a polynomial in $\phi$, for example.

This system apparently has not been studied before, which might be related to the fact that the action is Grassmann odd. However, in line with the present attempt to find a classical foundation of a quantum field theory, no path integral quantization (or other) of the model is intended, which could be obstructed by such a fermionic action.

Introducing canonical momenta,

$$
P_{\phi} \equiv \frac{\delta L}{\delta \dot{\phi}}=\dot{\psi}, \quad P_{\psi} \equiv \frac{\delta L}{\delta \dot{\psi}}=\dot{\phi},
$$

as usual, one calculates the Hamiltonian,

$$
\begin{aligned}
H & =\int \mathrm{d}^{3} x\left(P_{\phi} \dot{\phi}+P_{\psi} \dot{\psi}\right)-L \\
& =\int \mathrm{d}^{3} x\left(P_{\phi} P_{\psi}+\phi K \psi\right),
\end{aligned}
$$

which turns out to be Grassmann odd as well. Here the first of two useful abbreviations has been introduced: $K \equiv-\Delta+$ $m^{2}+v(\phi), K^{\prime} \equiv K+\phi \mathrm{d} v(\phi) / \mathrm{d} \phi$.

Hamilton's equations of motion for our model follow:

$$
\begin{aligned}
\dot{\phi} & =\frac{\delta H}{\delta P_{\phi}}=P_{\psi}, \\
\dot{\psi} & =\frac{\delta H}{\delta P_{\psi}}=P_{\phi}, \\
\dot{P}_{\phi} & =-\frac{\delta H}{\delta \phi}=-K^{\prime} \psi, \\
\dot{P}_{\psi} & =-\frac{\delta H}{\delta \psi}=-K \phi .
\end{aligned}
$$

Combining the equations, one obtains:

$$
\ddot{\phi}=-K \phi, \quad \ddot{\psi}=-K^{\prime} \psi,
$$

i.e., the generally nonlinear field equations, where there is only a parametric coupling between the fields $\phi$ and $\psi$, namely of the former to the latter.

These equations are invariant under the global symmetry transformation,

$$
\phi \longrightarrow \phi+\varepsilon \psi
$$

where $\varepsilon$ is an infinitesimal real parameter. Associated is the Noether charge:

$$
C_{1} \equiv \int \mathrm{d}^{3} x P_{\phi} \psi
$$

which is a constant of motion. Similarly, a second global symmetry transformation leaves the system invariant:

$$
\psi \longrightarrow \psi+\varepsilon \dot{\phi}
$$


with associated conserved Noether charge:

$$
C_{2} \equiv \int \mathrm{d}^{3} x\left(\frac{1}{2} P_{\psi}^{2}+V(\phi)\right)
$$

which is the total energy of the classical scalar field, with $\mathrm{d} V(\phi) / \mathrm{d} \phi \equiv K \phi$, appropriately taking care of gradient terms by partial integration.

In the following, it will be useful to introduce the Poisson bracket operation acting on two observables $A$ and $B$, which generally can be function(al)s of the phase space variables $\phi, P_{\phi}, \psi, P_{\psi}$ :

$$
\begin{aligned}
\{A, B\} \equiv A \int \mathrm{d}^{3} x & \left(\frac{\overleftarrow{\delta}}{\delta P_{\phi}} \frac{\vec{\delta}}{\delta \phi}+\frac{\overleftarrow{\delta}}{\delta P_{\psi}} \frac{\vec{\delta}}{\delta \psi}\right. \\
& \left.\leftarrow \frac{\delta}{\delta \phi} \frac{\vec{\delta}}{\delta P_{\phi}}-\frac{\overleftarrow{\delta}}{\delta \psi} \frac{\vec{\delta}}{\delta P_{\psi}}\right) B
\end{aligned}
$$

where all functional derivatives refer to the same space-time argument and act in the indicated direction; for the fermionic variables this direction is meant to coincide with their left/right-derivative character [18].

Note that $\{A, B\}=-\{B, A\}$, if the derivatives of $A$ and $B$ commute, i.e., if in each contributing term at least one of the two is Grassmann even. Furthermore, for any observable A, the usual relation among time derivatives holds:

$$
\frac{\mathrm{d}}{\mathrm{d} t} A=\{H, A\}+\partial_{t} A
$$

which embodies Hamilton's equations of motion.

Naturally, the time independent Hamiltonian of Eq. (4) is conserved by the evolution according to the classical equations of motion.

For the Hamiltonian and Noether charge densities, identified by $H \equiv \int \mathrm{d}^{3} x H(x)$ and $\left.C_{j} \equiv \int \mathrm{d}^{3} x C_{j}(x)\right|_{j=1,2}$, respectively, one finds a local (equal-time) supersymmetry algebra:

$$
\left\{C_{1}(x), C_{2}\left(x^{\prime}\right)\right\}=-H(x) \delta^{3}\left(x-x^{\prime}\right),
$$

and,

$$
\begin{aligned}
\left\{H(x), C_{1}\left(x^{\prime}\right)\right\}+ & \left\{C_{1}\left(x^{\prime}\right), H(x)\right\} \\
=\left\{H(x), C_{1}\left(x^{\prime}\right)\right\}+ & \left\{C_{1}(x), H\left(x^{\prime}\right)\right\}=0, \\
\left\{H(x), C_{2}\left(x^{\prime}\right)\right\}=0, & =0 .
\end{aligned}
$$

In all calculations, eventually arising coincidence limits are assumed to be smooth, since classical fields are involved. Of course, for any one of the constants of motion, $A \in$ $\left\{H, C_{1}, C_{2}\right\}$, one obtains: $\{H, A\}=\dot{A}=0$.

In the following section, the present analysis is applied to the corresponding phase space representation of an ensemble of systems and, furthermore, developed into an equivalent Hilbert space picture.

\section{THE LIOUVILLE EQUATION: FROM THE FIELD THEORY IN PHASE SPACE TO THE HILBERT SPACE PICTURE}

A particular example of Eq. (15) is the Liouville equation for a conservative system, such as the model considered in Section II. Considering an ensemble of systems, especially with some distribution over different initial conditions, this equation governs the evolution of its phase space density $\rho$ :

$$
0=i \frac{\mathrm{d}}{\mathrm{d} t} \rho=i \partial_{t} \rho-\hat{\mathcal{L}} \rho
$$

where a convenient factor $i$ has been introduced, and the Liouville operator $\hat{\mathcal{L}}$ is defined by:

$$
-\hat{\mathcal{L}} \rho \equiv i\{H, \rho\}
$$

These equations summarize the classical statistical mechanics of a conservative system, given the Hamiltonian $H$ in terms of the phase space variables.

Next, let us briefly recall the equivalent Hilbert space formulation developed by Koopman and von Neumann [2]. It will be modified here in a way appropriate for the supersymmetric classical field theory in question.

Two postulates are put forth:

- (A) the phase space density functional can be factorized in the form $\rho \equiv \Psi^{*} \Psi$;

- (B) the Grassmann valued and, in general, complex state functional $\Psi$ itself obeys the Liouville Eq. (20).

Furthermore, the complex valued inner product of such state functionals is defined by:

$$
\langle\Psi \mid \Phi\rangle \equiv \int \mathcal{D} \phi \mathcal{D} P_{\psi} \mathcal{D} \psi \mathcal{D} P_{\phi} \Psi^{*} \Phi=\langle\Phi \mid \Psi\rangle^{*}
$$

i.e., by functional integration over all phase space variables (fields). However, due to the presence of Grassmann valued variables, the $*$-operation which defines the dual of a state functional needs special attention and will be discussed shortly.

The above definitions make sense for functionals which suitably generalize the notion of square-integrable functions. In particular, the functional integrals can be treated rigorously by discretizing the system, properly pairing degrees of freedom.

Given the Hilbert space structure, the Liouville operator of a conservative system has to be Hermitian and the overlap $\langle\Psi \mid \Psi\rangle$ is a conserved quantity. Then, the Liouville equation also applies to $\rho=|\Psi|^{2}$, due to its linearity, and $\rho$ may be interpreted as replacing the probability density of before [2]. Naturally, this is needed for meaningful phase space expectation values of observables.

Certainly, one is reminded here of the usual quantum mechanical formalism. In order to expose the striking similarity as well as the remaining crucial difference, further transformations of the functional Liouville equation are useful [1]. 
A Fourier transformation replaces the momentum $P_{\psi}$ by a second scalar field $\bar{\phi}$. Furthermore, define $\bar{\psi} \equiv P_{\phi}$. Thus, the Eqs. (20)-(21) yield:

$$
i \partial_{t} \Psi=\hat{\mathcal{H}} \Psi
$$

where $\Psi$ is considered as a functional of $\phi, \bar{\phi}, \psi, \bar{\psi}$, and with the emergent "Hamilton operator":

$$
\begin{aligned}
& \hat{\mathcal{H}} \Psi \equiv-i \int \mathcal{D} P_{\Psi} \exp \left(i P_{\psi} \cdot \bar{\phi}\right)\{H, \Psi\} \\
= & \int \mathrm{d}^{3} x\left(-\delta_{\bar{\phi}} \delta_{\phi}+\bar{\phi} K \phi-i\left(\bar{\psi} \delta_{\psi}-\psi K^{\prime} \delta_{\bar{\psi}}\right)\right) \Psi \\
\equiv & \int \mathrm{d}^{3} x \hat{\mathcal{H}}(x) \Psi
\end{aligned}
$$

using the abbreviation $f \cdot g \equiv \int \mathrm{d}^{3} x f(x) g(x)$. Note that the density $\hat{\mathcal{H}}(x)$ is Grassmann even.

While the Eq. (23) strongly resembles a functional Schrödinger equation, several comments must be made here which point out its different character.

First of all, following a linear transformation of the scalar field variables, $\phi \equiv(\sigma+\kappa) / \sqrt{2}$ and $\bar{\phi} \equiv(\sigma-\kappa) / \sqrt{2}$, one finds a "bosonic" kinetic energy term:

$$
-\frac{1}{2} \int \mathrm{d}^{3} x\left(\delta_{\sigma}^{2}-\delta_{\kappa}^{2}\right)
$$

which is not bounded from below. Therefore, neglecting the Grassmann variables momentarily, the remaining Hermitian part of the Hamiltonian lacks a lowest energy state, which otherwise could qualify as the emergent quantum mechanical groundstate of the bosonic sector.

Secondly, as could be expected, the fermionic sector reveals a similar problem.

The *-operation mentioned before amounts to complex conjugation for a bosonic state functional, $(\Psi[\bar{\phi}, \phi])^{*} \equiv$ $\Psi^{*}[\bar{\phi}, \phi]$, analogously to an ordinary wave function in quantum mechanics. However, based on complex conjugation alone, the fermionic part of the Hamiltonian (24) would not be Hermitian.

Instead, a detailed construction of the inner product for functionals of Grassmann valued fields has been presented in Ref. [21]; see also further examples in Refs. [22]. Considering only the noninteracting case with $K^{\prime}=K$, i.e., with $v(\phi)=0$ in Eq. (2), the construction of Floreanini and Jackiw can be directly applied here. Then, the Hermitian conjugate of $\psi$ is $\psi^{\dagger}=\delta_{\psi}$ and of $\bar{\psi}$ it is $\bar{\psi}^{\dagger}=\delta_{\bar{\psi}}$. Furthermore, rescaling $\bar{\psi} \longrightarrow \bar{\psi} \sqrt{K}$, the fields $\bar{\psi}$ and $\psi$ obtain the same dimensionality. Together, this suffices to render Hermitian the fermionic part of the Hamiltonian (24), which becomes:

$$
\hat{\mathcal{H}}_{\bar{\psi} \psi} \equiv i\left(\psi \sqrt{K} \delta_{\bar{\psi}}-\bar{\psi} \sqrt{K} \delta_{\psi}\right)
$$

In the presence of interactions, with $K^{\prime} \neq K$, additional modifications are necessary and will be considered elsewhere. In any case, although $\hat{\mathcal{H}}_{\bar{\psi} \psi}$ must be (made) Hermitian, its eigenvalues generally will not have a lower bound either.
To summarize, the emergent Hamiltonian $\hat{\mathcal{H}}$ tends to be unbounded from below, thus lacking a groundstate. This generic difficulty has been encountered in various attempts to build deterministic quantum models, i.e., classical models which can simultaneously be seen as quantum mechanical ones $[3,6,7,9,10]$. For the present case, this will be discussed and resolved in Section IV.

To conclude this section, equal-time operator relations for the interacting case are derived here, which are related to the supersymmetry algebra of Eqs. (16)-(19). This is achieved by Fourier transformation of appropriate Poisson brackets, similarly as with the emergent Hamiltonian in Eq. (24) above.

To begin with, the operators corresponding to the Noether densities will be useful. Using Eq. (11) and $\bar{\psi} \equiv P_{\phi}$, as before, one obtains:

$$
\begin{aligned}
\hat{\mathcal{C}}_{1}(x) \Psi & \equiv \int \mathcal{D} P_{\Psi} \exp \left(i P_{\psi} \cdot \bar{\phi}\right)\left\{C_{1}(x), \Psi\right\} \\
& =\left(-\psi \delta_{\phi}+i \bar{\psi} \bar{\phi}\right)_{(x)} \Psi
\end{aligned}
$$

Similarly, one obtains:

$$
\hat{\mathcal{C}}_{2}(x) \Psi \equiv\left(-i \delta_{\bar{\phi}} \delta_{\psi}-\phi K \delta_{\bar{\psi}}\right)_{(x)} \Psi
$$

which is related to Eq. (13).

Both operators are Grassmann odd and obey:

$$
\left\{\hat{\mathcal{C}}_{j}(x), \hat{\mathcal{C}}_{j}\left(x^{\prime}\right)\right\}_{+}=0
$$

for $j=1,2$. Therefore, they are nilpotent, $\hat{C}_{j}^{2}(x)=0$. This should be compared to Eq. (19), as well as the vanishing commutator:

$$
\left[\hat{\mathcal{H}}(x), \hat{\mathcal{H}}\left(x^{\prime}\right)\right]=0
$$

where $[\hat{A}, \hat{B}] \equiv \hat{A} \hat{B}-\hat{B} \hat{A}$. Thus, the emergent theory is local, as expected.

It should be remarked that in all calculations of (anti)commutation relations eventually necessary partial integrations, i.e. shifting of gradients, are justified by smearing with suitable test functions and integrating.

Further relations that correspond to Jacobi identities on the level of the Poisson brackets are interesting. Generally, one has to be careful about extra signs that arise due to the Grassmann valued quantities, as compared to more familiar ones related to real or complex variables [18]. Straightforward calculation gives:

$$
\begin{aligned}
{\left[\hat{\mathcal{H}}(x), \hat{\mathcal{C}}_{j}\left(x^{\prime}\right)\right] } & =0, \text { for } j=1,2, \\
\left\{i \hat{\mathcal{C}}_{1}(x), \hat{\mathcal{C}}_{2}\left(x^{\prime}\right)\right\}_{+} & =\hat{\mathcal{H}}(x) \delta^{3}\left(x-x^{\prime}\right),
\end{aligned}
$$

cf. Eqs. (16)-(18); the extra factor $i$ must be attributed to the Fourier transformation that enters between the phase space functions before and the operators here.

Finally, it is noteworthy that a copy of the above operator algebra arises, if one performs the replacements $\psi \leftrightarrow-\delta_{\bar{\psi}}$ and $\bar{\psi} \leftrightarrow \delta_{\psi}$ on the operators $\hat{\mathcal{C}}_{j}$. This yields the nilpotent 
operators $\hat{\mathcal{D}}_{j}$, instead of the $\hat{\mathcal{C}}_{j}$ :

$$
\begin{aligned}
i \hat{\mathcal{D}}_{1}(x) & \equiv\left(i \delta_{\bar{\psi}} \delta_{\phi}-\delta_{\psi} \bar{\phi}\right)_{(x)}, \\
\hat{\mathcal{D}}_{2}(x) & \equiv\left(i \delta_{\bar{\phi}} \bar{\psi}-\phi K \psi\right)_{(x)},
\end{aligned}
$$

with a convenient overall sign introduced in the latter definition. They fullfill the same (anti)commutation relations as in Eqs. (30)-(33).

Finally, also the following local operators commute with the Hamiltonian density:

$$
\left(i \hat{\mathcal{D}}_{1} \hat{\mathcal{C}}_{2} \pm i \hat{\mathcal{C}}_{1} \hat{\mathcal{D}}_{2}\right)=-i\left(\delta_{\bar{\psi}} \delta_{\psi} \mp \bar{\psi} \psi\right)\left(-\delta_{\bar{\phi}} \delta_{\phi}+\bar{\phi} K \phi\right)
$$

with $\left[\hat{\mathcal{C}}_{1}, \hat{\mathcal{D}}_{2}\right]=\left[\hat{\mathcal{D}}_{1}, \hat{\mathcal{C}}_{2}\right]=0$. These operators are not nilpotent. Their square, though, is highly singular.

One may complete these considerations with the full set of operators generating the ordinary space-time symmetries of our model. However, they are not believed to play a special role for the considerations of the following section. There, the no-groundstate problem of the emergent Hamiltonian, Eq. (24), will be addressed.

\section{PROVIDING THE GROUNDSTATE OF THE EMERGENT QUANTUM MODEL}

Following Eq. (24), it has been pointed out that the emergent Hamiltonian lacks a proper groundstate, i.e., its spectrum is not bounded from below. This prohibits to interpret the model, as it stands, as a quantum mechanical one already, despite close formal similarities.

In order to overcome this difficulty, the general strategy is to find a positive definite local operator $\hat{P}$ that commutes with the Hamiltonian density, $\left[\hat{\mathcal{H}}(x), \hat{P}\left(x^{\prime}\right)\right]=0$. Then, the Hamiltonian can be split into contributions with positive and negative spectrum:

$$
\hat{\mathcal{H}}=\hat{\mathcal{H}}_{+}-\hat{\mathcal{H}}
$$

where:

$$
\hat{\mathcal{H}}_{ \pm} \equiv \int \mathrm{d}^{3} x F(\hat{\mathcal{H}}(x) \pm \hat{P}(x)) .
$$

Here $F$ can be any even function with the property:

$$
F(a+b)-F(a-b)=a b G\left(a^{2}, b^{2}\right), G>0,
$$

for $a, b \in \mathbf{R}$.

The simplest example is $F(a) \equiv a^{2}, G \equiv 4$. With this, the splitting of $\hat{\mathcal{H}}$ is explicitly given by:

$$
\hat{\mathcal{H}}=\int \mathrm{d}^{3} x\left(\frac{(\hat{\mathcal{H}}+\hat{P})^{2}-(\hat{\mathcal{H}}-\hat{P})^{2}}{4 \hat{P}}\right)
$$

i.e., $\hat{\mathcal{H}}_{ \pm}(x)=(\hat{\mathcal{H}}(x) \pm \hat{P}(x))^{2} / 4 \hat{P}(x)$. A quartic polynomial could be used instead, etc. In the absence of further symmetry requirements, or other, from the model under consideration, the simplest splitting will do. It will allow us to obtain a free quantum field theory, in particular, as leading part of the relevant Hamilton operator.

Here, as in the following, a regularization is necessary, in order to give a meaning particularly to some of the squared operators that will keep appearing.

Finally, the spectrum of the Hamiltonian $\hat{\mathcal{H}}$ is made bounded from below by imposing the "positivity constraint":

$$
\hat{\mathcal{H}} \Psi=0
$$

This constraint can be enforced as an initial condition, for example, and is preserved by the evolution, since $\left[\hat{\mathcal{H}}_{+}(x), \hat{\mathcal{H}}(x)\right]=0$, by construction. In this way, the physical states of the system are selected which are based on the existence of a quantum mechanical groundstate.

Such a constraint selecting the physical part of the emergent Hilbert space has been earlier discussed in the models of Refs. [3, 9, 10]. It has been interpreted by 't Hooft as "information loss" at the fundamental level where quantum mechanics may arise from a deterministic theory. However, it seems also quite possible to relate this to a dynamical symmetry breaking phenomenon instead, cf. Section V.

For our field theory, the noninteracting and interacting cases shall now be studied separately in more detail.

\section{A. The noninteracting case}

As mentioned before, with $v(\phi)=0$ in Eq. (2), and therefore $K^{\prime}=K=-\Delta+m^{2}$, the rescaling $\bar{\psi} \longrightarrow \bar{\psi} \sqrt{K}$ is useful, and one may consider the set of operators:

$$
\begin{aligned}
\hat{\mathcal{H}}(x) & =\left(-\delta_{\bar{\phi}} \delta_{\phi}+\bar{\phi} K \phi\right)_{(x)}+\hat{\mathcal{H}}_{\bar{\psi} \psi}(x), \\
i \hat{\mathcal{C}}_{1}(x) & =\left(-i \psi \delta_{\phi}-\bar{\psi} \sqrt{K} \bar{\phi}\right)_{(x)}, \\
\hat{\mathcal{C}}_{2}(x) & =\left(-i \delta_{\bar{\phi}} \delta_{\psi}-\phi \sqrt{K} \delta_{\bar{\psi}}\right)_{(x)},
\end{aligned}
$$

with $\hat{\mathcal{H}}_{\bar{\psi} \psi}$ from Eq. (27). These operators fullfill the same operator algebra as discussed in the previous section.

Furthermore, let us consider the Hermitian conjugate operators, in this case based on $\psi^{\dagger}=\delta_{\psi}$ and $\bar{\psi}^{\dagger}=\delta_{\bar{\psi}}[21]$ :

$$
\begin{aligned}
\left(i \hat{\mathcal{C}}_{1}(x)\right)^{\dagger} & =\left(-i \delta_{\psi} \delta_{\phi}-\delta_{\bar{\psi}} \sqrt{K} \bar{\phi}\right)_{(x)} \\
\left(\hat{\mathcal{C}}_{2}(x)\right)^{\dagger} & =\left(-i \delta_{\bar{\phi}} \psi-\phi \sqrt{K} \bar{\psi}\right)_{(x)}
\end{aligned}
$$

They commute with the Hermitian density $\hat{\mathcal{H}}(x)$, and one finds that $\left\{i \hat{\mathcal{C}}_{1}(x),\left(\hat{\mathcal{C}}_{2}(x)\right)^{\dagger}\right\}_{+}=0$, together with the corresponding adjoint relation.

Then, also the following Hermitian operators commute 
with the Hamiltonian density:

$$
\begin{aligned}
& \hat{\mathcal{C}}_{1+}(x) \equiv\left(i \hat{\mathcal{C}}_{1}(x)+\left(i \hat{\mathcal{C}}_{1}(x)\right)^{\dagger}\right) \sqrt{2} \\
& =\frac{1}{\sqrt{2}}\left(-i\left(\delta_{\psi}+\psi\right) \delta_{\phi}-\left(\delta_{\bar{\psi}}+\bar{\psi}\right) \sqrt{K} \bar{\phi}\right)_{(x)}, \\
& \hat{\mathcal{C}}_{2+}(x) \equiv\left(\hat{\mathcal{C}}_{2}(x)+\left(\hat{\mathcal{C}}_{2}(x)\right)^{\dagger}\right) / \sqrt{2} \\
& =\frac{1}{\sqrt{2}}\left(-i\left(\delta_{\psi}+\psi\right) \delta_{\bar{\phi}}-\left(\delta_{\bar{\psi}}+\bar{\psi}\right) \sqrt{K} \phi\right)_{(x)} .
\end{aligned}
$$

These operators are particularly interesting, since they present, in some sense, the "square-root of the harmonic oscillator":

$$
\begin{aligned}
& \hat{\mathcal{C}}_{1+}^{2}(x)=\frac{\delta^{3}(0)}{2}\left(-\delta_{\phi}^{2}+\bar{\phi} K \bar{\phi}\right)_{(x)}, \\
& \hat{\mathcal{C}}_{2+}^{2}(x)=\frac{\delta^{3}(0)}{2}\left(-\delta_{\bar{\phi}}^{2}+\phi K \phi\right)_{(x)},
\end{aligned}
$$

or, rather, since their sum amounts to the Hamiltonian density of two free bosonic quantum fields.

It seems natural now to choose the positive definite local operator $\hat{P}$ of Eq. (40) as:

$$
\hat{P}(x) \equiv \frac{\xi}{\delta^{3}(0)}\left(\hat{\mathcal{C}}_{1+}^{2}(x)+\hat{\mathcal{C}}_{2+}^{2}(x)\right)
$$

where $\xi$ is a dimensionless parameter. This results in the operators of definite sign:

$$
\begin{aligned}
\hat{\mathcal{H}}_{ \pm}(x)= & (\hat{\mathcal{H}}(x) \pm \hat{P}(x))^{2} / 4 \hat{P}(x) \\
= & \frac{\xi}{8}\left(-\delta_{\phi}^{2}+\phi K \phi-\delta_{\bar{\phi}}^{2}+\bar{\phi} K \bar{\phi}\right) \\
& \pm \frac{1}{2} \hat{\mathcal{H}}(x)+\frac{1}{4} \hat{\mathcal{H}}^{2}(x) / \hat{P}(x)
\end{aligned}
$$

cf. Eqs. (37)-(40).

Setting $\xi=2$ and performing again the linear transformation $\phi \equiv(\sigma+\kappa) / \sqrt{2}$ and $\bar{\phi} \equiv(\sigma-\kappa) / \sqrt{2}$, previously mentioned after Eqs. (24)-(25), here instead yields the Hamiltonian density:

$$
\hat{\mathcal{H}}_{+}(x)=\frac{1}{2}\left(-\delta_{\sigma}^{2}+\sigma K \sigma+\hat{\mathcal{H}}_{\bar{\psi} \psi}+\frac{1}{2} \hat{\mathcal{H}}^{2} / \hat{P}\right)_{(x)},
$$

with $\hat{\mathcal{H}}_{\bar{\psi} \psi}$ from Eq. (27), and where, of course, the linear transformation has also been performed in $\hat{\mathcal{H}}^{2} / \hat{P}$. One observes that the only trace of the previous instability is now relegated to this last term, which still involves the scalar field $\kappa$. The $l o-$ cal interactions present in this term certainly have a nonstandard form. Additional parameters playing the role of coupling constants could be introduced by a more complicated splitting of the emergent Hamiltonian, see Eqs. (37)-(40), or a different choice for the operator $\hat{P}$.

However, the Hamilton operator $\hat{\mathcal{H}}_{4}$ has a positive spectrum, by construction, and the leading terms are those of a free bosonic quantum field together with a fermion doublet in the Schrödinger representation. They dominate at low energy.

Similarly, the constraint operator density becomes:

$$
\hat{\mathcal{H}}(x)=\frac{1}{2}\left(-\delta_{\kappa}^{2}+\kappa K \kappa-\hat{\mathcal{H}}_{\bar{\psi} \psi}+\frac{1}{2} \hat{\mathcal{H}}^{2} / \hat{P}\right)_{(x)} .
$$

A certain symmetry with Eq. (53) is obvious; note that $-\hat{\mathcal{H}}_{\bar{\psi} \psi}=\hat{\mathcal{H}}_{\psi \bar{\psi}}$. It suggests to think of the elimination of part of the Hilbert space, Eq. (41), as a dynamical symmetry breaking effect. This point will be briefly addressed in the concluding section.

\section{B. The interacting case}

In the interacting case, one has $v(\phi) \neq 0$ in Eq. (2), $K \equiv$ $-\Delta+m^{2}+v(\phi)$, and $K^{\prime} \equiv K+\phi \mathrm{d} v(\phi) / \mathrm{d} \phi$. While the operator algebra of Section III is available, it is difficult to find the corresponding generalization of the "square-root of the harmonic oscillator" operators of Eqs. (47)-(48).

The latter were most useful, however, in order to obtain a positive definite operator $\hat{P}$ that commutes with the emergent Hamiltonian $\hat{\mathcal{H}}$ and, with this, to achieve its splitting into parts with positive and negative spectrum, as in Eqs. (37)-(40). The vanishing commutator here is important, since it assures that this splitting is invariant under evolution of the system.

Furthermore, said operators are particularly interesting, if the resulting bounded Hamilton operator $\hat{\mathcal{H}}_{4}$ is to contain leading standard field theory terms, even though modified by additions as in Eq. (53), for example.

Following these remarks, one could try and construct such operators perturbatively, i.e., by deforming the operators, and include step by step increasing orders in the interaction $v$.

A quite different approach might be to choose:

$$
\hat{P}(x) \equiv \frac{1}{\mathcal{M}} \hat{\mathcal{H}}^{2}(x)
$$

where $\mathcal{M}$ is a parameter with dimensions of energy per unit volume. (Note that replacing the Hamiltonian density squared with the total angular momentum density squared would introduce a constant with dimensions of action per unit area.) This operator commutes with the Hamiltonian density and will lead to a positive $\hat{\mathcal{H}}_{4}$. In fact, the resulting contributions to the Hamiltonian are in this case simply given by:

$$
\hat{\mathcal{H}}_{ \pm}=\frac{1}{4 \mathcal{M}} \int \mathrm{d}^{3} x(\mathcal{M} \pm \hat{\mathcal{H}}(x))^{2} .
$$

Now, imposing the constraint, $\hat{\mathcal{H}} \Psi=0$, one finds that on physical states the bounded Hamilton operator gives:

$$
\hat{\mathcal{H}}_{4} \Psi=\frac{1}{\mathcal{M}} \int \mathrm{d}^{3} x \hat{\mathcal{H}}^{2}(x) \Psi=\mathcal{M} \cdot \mathcal{V} \Psi
$$

with $\mathcal{V} \equiv \int \mathrm{d}^{3} x$. A surprisingly restrictive result.

To be sure, if one wants to connect the Hamilton operator $\hat{\mathcal{H}}_{4}$ of Eq. (57) to familiar quantum field theories, the difficult 
task of finding "square-root of the harmonic oscillator" operators reappears. Here one has to find an underlying classical model for which the emergent Hamiltonian $\hat{\mathcal{H}}$, cf. Eqs. (24)(25), contains terms which are linear in such operators.

\section{CONCLUSIONS}

The work presented here touches a number of conceptual issues surrounding quantum theory. The interpretation of the measurement process and of the "collapse of the wave function", in particular, must figure prominently in this context, together with the "quantum indeterminism" and the wider philosophical implications of the algorithmic rules comprising quantum theory as a whole [23]. It is left for future studies to find out, how a deterministic framework, such as further elaborated here, allows to see them in a new light.

Deterministic models which simultaneously and consistently can be described as quantum mechanical ones present a challenge to common wisdom concerning the meaning, foundations, and limitations of quantum theory. Main aspects of the present work on such a model taken from field theory can be summarized as follows.

A fairly standard description of the dynamics in phase space and its conversion to an operators-in-Hilbert-space formalism à la Koopman and von Neumann [2] yield a wave functional equation which is surprisingly similar to the functional Schrödinger equation of quantum field theory. However, the emergent "Hamilton operator" of this picture, generically, lacks a groundstate, which corresponds to the spectrum not being bounded from below. In order to arrive at a proper quantum theory with a stable groundstate, parts of the Hilbert space have to be removed by a positivity constraint which is preserved by the Hamiltonian flow.

In the present example, this has been discussed based on simple supersymmetry properties of the underlying classical model. The important role of "square-root of the harmonic oscillator" operators in constructing the constraint operator has been pointed out, and they have been constructed in the limit of classically noninteracting scalar and fermionic fields, the latter being represented by nilpotent Grassmann valued variables. Several comments on the interacting case have been made, where they may be constructed in perturbation theory. In particular, these operators promise to be important in emergent quantum models that smoothly connect to standard field theories with leading quadratic kinetic energy terms.

Here I should like to conclude with a more speculative remark concerning the dynamical origin of the positivity constraint, which has been introduced and interpreted as a "loss of information" at the fundamental dynamical level earlier $[3,9,10]$. The latter anticipates a still unknown, possibly dissipative information loss mechanism in the classical theory beneath, such as due to an unavoidable coarse-graining in the description of some deterministic chaotic dynamics. This would turn the system under study into an open system.

However, the discussion in Section IV indicates a complementary point of view. There is a great deal of symmetry between the operators $\hat{\mathcal{H}}_{4}$ and $\hat{\mathcal{H}}$ which are responsible for the evolution of the system as well as for the selection of the physical states. In fact, since the emergent functional wave equation is linear in the time derivative, positive and negative parts of the spectrum of the emergent Hamiltonian $\hat{\mathcal{H}}$, see Eqs. (24)-(25), can be turned into each other by reversing the direction of time. Correspondingly, the roles of $\hat{\mathcal{H}}_{4}$ and $\hat{\mathcal{H}}$ can be exchanged.

This suggests that giving preference to one over the other in determining the physical states may be a contingent property of the system. It typically occurs in situations where a symmetry is dynamically broken.

Let us consider an extension of the present model which schematically incorporates such an effect. Introducing a local "order parameter" $\hat{O}$, take the new Hamilton operator density:

$$
\hat{\mathcal{H}}_{*}(x) \equiv \hat{\mathcal{H}}_{+}(x)-\hat{\mathcal{H}}(x) \tanh \hat{O}(x)
$$

with $\left[\hat{\mathcal{H}}_{ \pm}(x), \hat{O}\left(x^{\prime}\right)\right]=0$ and, for example, $\hat{O} \equiv(\hat{P}-\mathcal{M}) / \mathcal{M}$ or $\hat{O} \equiv\left(\hat{\mathcal{H}}^{2}-\mathcal{M}^{2}\right) / \mathcal{M}^{2}$. The positive operators $\hat{\mathcal{H}}_{ \pm}$are as defined in Eqs. (37)-(40), $\hat{P}$ is positive definite, cf. Section IV, and $\mathcal{M}$ denotes an energy density parameter. All operators here commute.

Therefore, the eigenstates can be ordered according to the eigenvalues of $\hat{\mathcal{H}}=\hat{\mathcal{H}}+\hat{\mathcal{H}}$ or $\hat{P}$.

For large values of the order parameter, at high energy, loosely speaking, the symmetry is restored and asymptotically $\hat{\mathcal{H}}_{*} \approx \hat{\mathcal{H}}_{+}-\hat{\mathcal{H}}$. In this regime, the system behaves classically, corresponding to an emergent Hamilton operator with unbounded spectrum. Here, the role of $\hat{\mathcal{H}}_{4}$ and $\hat{\mathcal{H}}$ could approximately be interchanged by changing the direction of time.

Conversely, for small values of the order parameter, one qualitatively finds $\hat{\mathcal{H}}_{*} \approx \hat{\mathcal{H}}+\hat{\mathcal{H}} \tanh (1)=\left(\hat{P}+\hat{\mathcal{H}}^{2} / \hat{P}\right) / 2 \geq$ 0 . This result should be compared with Eqs. (51)-(54), for example, and particularly with Eq. (53). Here the spectrum of $\hat{\mathcal{H}}_{*}$ is bounded from below and the system behaves quantum mechanically. Interestingly, the backbending of the negative branch of the spectrum to positive values has replaced the imposition of the positivity constraint, Eq. (41).

The precise nature of the transition between classical and quantum regimes, which is regulated by the parameter $\mathcal{M}$, depends on how and which order parameter comes into play. Due to its nonlinearity, which introduces higher order functional derivatives, it modifies the underlying phase space dynamics, see Eqs. (20)-(24). It will be interesting to further study such corrections, which must contribute as additional force terms, depending on higher powers of field momentum, for example, to the classical Liouville operator.

Differently from a possible "loss of information" mechanism, presently all operators involved are Hermitian and closely related to the symmetry properties of the system.

Such a symmetry breaking mechanism might be responsible for the emergent quantization also in other cases than the (pseudo)classical field theory presented here. Besides this, models that incorporate interacting fermions and gauge fields are an important topic for future study. Furthermore, time reparametrization or general diffeomorphism invariance 
should naturally be most interesting to consider in the framework of deterministic quantum models.

\section{Acknowledgements}

I wish to thank A. DiGiacomo for many discussions and kind hospitality at the Dipartimento di Fisica in Pisa and G. Vitiello, M. Blasone, L. Lusanna, E. Sorace, and M. Ciafaloni for helpful remarks and discussions during visits at both, the Dipartimento di Fisica in Salerno and in Firenze.
[1] H.-T. Elze, Phys. Lett. A 335, 258 (2005)

[2] B.O. Koopman, Proc. Nat. Acad. Sci. (USA) 17, 315 (1931); J. von Neumann, Ann. Math. 33, 587 (1932); ibid. 33, 789 (1932).

[3] G. 't Hooft, J. Stat. Phys. 53, 323 (1988); Quantum Mechanics and Determinism, in: Proc. of the Eighth Int. Conf. on "Particles, Strings and Cosmology", ed. by P. Frampton and J. Ng (Rinton Press, Princeton, 2001), p. 275; hep-th/0105105 ; see also: Determinism Beneath Quantum Mechanics, quant$\mathrm{ph} / 0212095$

[4] A. Einstein, B. Podolsky, and N. Rosen, Phys. Rev. 47, 777 (1935).

[5] J.S. Bell, "Speakable and Unspeakable in Quantum Mechanics" (Cambridge U. Press, Cambridge, 1987).

[6] H.-T. Elze and O. Schipper, Phys. Rev. D 66, 044020 (2002); H.-T. Elze, Phys. Lett. A 310, 110 (2003).

[7] H.-T. Elze, Physica A 344, 478 (2004); Quantum Mechanics and Discrete Time from "Timeless" Classical Dynamics, in: Ref. [11], p. 196; quant-ph/0306096.

[8] E. Gozzi, M. Reuter, and W.D. Thacker, Phys. Rev. D 40, 3363 (1989); D 46, 757 (1992).

[9] M. Blasone, P. Jizba, and G. Vitiello, Phys. Lett. A 287, 205 (2001); M. Blasone, E. Celeghini， P. Jizba, and G. Vitiello, Phys. Lett. A 310, 393 (2003).

[10] M. Blasone, P. Jizba, and H. Kleinert, Phys. Rev. A, in press, quant-ph/0409021; Braz. J. Phys. 35, 497 (2005).

[11] "Decoherence and Entropy in Complex Systems", ed. by H.T. Elze, Lecture Notes in Physics, Vol. 633 (Springer-Verlag, Berlin Heidelberg New York, 2004).

[12] L. Smolin, Matrix Models as Non-Local Hidden Variables Theories, hep-th/0201031 ; F. Markopoulou and L. Smolin, Quantum Theory from Quantum Gravity, gr-qc/0311059.
[13] S.L. Adler, Quantum Mechanics as an Emergent Phenomenon: The Statistical Dynamics of Global Unitary Invariant Matrix Models as the Precursors of Quantum Field Theory (Cambridge U. Press, Cambridge, 2005).

[14] E. Nelson, Phys. Rev. 150, 1079 (1966).

[15] G. Parisi and Y.S. Wu, Sci. Sin. 24, 483 (1981); P.H. Damgaard and H. Hüffel, Phys. Rep. 152, 227 (1987).

[16] Closer inspection shows that the constraint considered in Ref. [1] is not sufficient to turn the classical model there into a quantum mechanical one. Presently, this is solved differently in Section IV.

[17] R. Casalbuoni, Nuovo Cim. 33A, 389 (1976); F.A. Berezin and M.S. Marinov, Ann. Phys. (NY) 104, 336 (1977).

[18] P.G.O. Freund, "Introduction to Supersymmetry" (Cambridge U. Press, Cambridge, 1986); B.DeWitt, "Supermanifolds", 2nd ed. (Cambridge U. Press, Cambridge, 1992).

[19] N.S. Manton， J. Math. Phys. 40， 736 (1999); G. Junker, S. Matthiesen, and A.Inomata, Classical and quasiclassical aspects of supersymmetric quantum mechanics, hep-th/95102230.

[20] Grassmann algebras and analysis over supernumbers are presented in detail by DeWitt [18]

[21] R. Floreanini and R. Jackiw, Phys. Rev. D 37, 2206 (1988).

[22] C. Kiefer and A.Wipf, Ann. Phys. (NY) 236, 241 (1994); A. Duncan, H. Meyer-Ortmanns, and R. Roskies, Phys. Rev. D 36, 3788 (1987).

[23] "Quantum Theory and Beyond", ed. by T. Bastin (Cambridge U. Press, Cambridge, 1971); "Quantum Theory and Measurement", ed. by J.A. Wheeler and W.H. Zurek (Princeton U. Press, Princeton, 1980). 